\title{
Atrial Bigeminy Associated With Hypothyroidism: A Case Report
}

\author{
Antonia Digklia ${ }^{\mathrm{a}}$, Ioannis A. Voutsadakis ${ }^{\mathrm{a}, \mathrm{b}}$
}

\begin{abstract}
The effect of hypothyroidism on the cardiovascular system has been well documented. Hypothyroidism produces both systolic and diastolic dysfunction that can lead to cardiac arrhythmia and congestive heart failure. The consequences of acute hypothyroidism due to thyroid hormone withdrawal in previously thyroidectomized patients on cardiac function have been investigated only in a few studies. We describe a 65 -year-old man, having previously undergone a thyroidectomy for thyroid cancer, in whom withdrawal of thyroid hormone led to cardiac arrhythmia manifested as bigeminy. It is the first report in the English literature where a short term overt hypothyroidism is associated with atrial bigeminy.
\end{abstract}

Keywords: Hypothyroidism; Cardiac arrhythmia; Atrial bigeminy; Thyroid cancer; Papillary

\section{Introduction}

Thyroid hormones have well described effects in the cardiovascular system. These include increased cardiac inotropy and chronotropy leading to increased cardiac output and decreased systemic vascular resistance [1]. Thus, hyperthyroidism produces sinus tachycardia and cardiac hyper-excitability that can be manifested as atrial fibrillation, atrial flutter or

\section{Manuscript accepted for publication April 4, 2011}

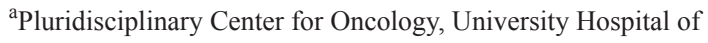
Lausanne, Lausanne, Switzerland

${ }^{\mathrm{b}}$ Corresponding author: Ioannis A. Voutsadakis, Pluridisciplinary

Center for Oncology, BH06, University Hospital of

Lausanne, Bugnon 46, Lausanne 1011, Switzerland.

Email: ivoutsadakis@yahoo.com

doi:10.4021/jmc203w increased premature atrial beats and ventricular extra-systoles $[1,2]$. In addition, because decreased peripheral vascular resistance cannot compensate for the increased heart rate, hypertension is often observed. In contrast, hypothyroidism produces sinus bradycardia and decreased cardiac contractility [3]. Paradoxically, this decreased contractility can lead to prolonged QT interval, heart blocks and other arrhythmias $[4,5]$.

\section{Case Report}

A 65-year-old man with papillary thyroid carcinoma of right lobe, stage T4 N1 M0, was initially treated with total thyroidectomy and postoperative radioiodine in two occasions 3 and 9 months after surgery. Thyroid hormone substitution was begun thereafter. A recurrence with ipsilateral regional lymph node metastases 1.5 years later was treated with right cervical lymphadenectomy and a third radioiodine administration. Nine years after the initial diagnosis, a follow-up $I^{131}$ scan showed small residual cervical thyroid tissue corresponding to a right para-laryngeal metastatic deposit. Patient underwent surgical excision of this metastasis and was planned for a fourth $\mathrm{I}^{131}$ therapy. He was prepared by discontinuation of his thyroid replacement 3 weeks before hospitalization and received recombinant human thyrotropin alpha ( $\mathrm{hTSH} \alpha)$ two days preceding his hospitalization.

Past medical history was unremarkable. Medications included valsartan for hypertension. He reported fatigue and decreased exercise tolerance for the past 4 weeks, but denied dyspnea, chest pain or palpitations.

Physical examination demonstrated a heart rate of 60 / min with a regularly irregular rhythm and a blood pressure of $117 / 78 \mathrm{mmHg}$.

Laboratory studies were significant for a TSH elevated at $57 \mu \mathrm{g} / \mathrm{ml}$ (normal $0.2-3.5 \mu \mathrm{g} / \mathrm{ml}$ ), a T4 of $<5 \mathrm{pmol} / 1$ (normal $9-19 \mathrm{pmol} / \mathrm{l})$ and thyroglobuline of $239 \mu \mathrm{g} / \mathrm{l}($ normal < $35 \mu \mathrm{g} / \mathrm{l})$.

The ECG (Fig. 1) showed two foci of depolarization originating in the atrium as evidenced by two different $p$ wave morphologies (A $p$ wave originating from the sinus has an initial elevation followed by oppression while a $p$ wave 


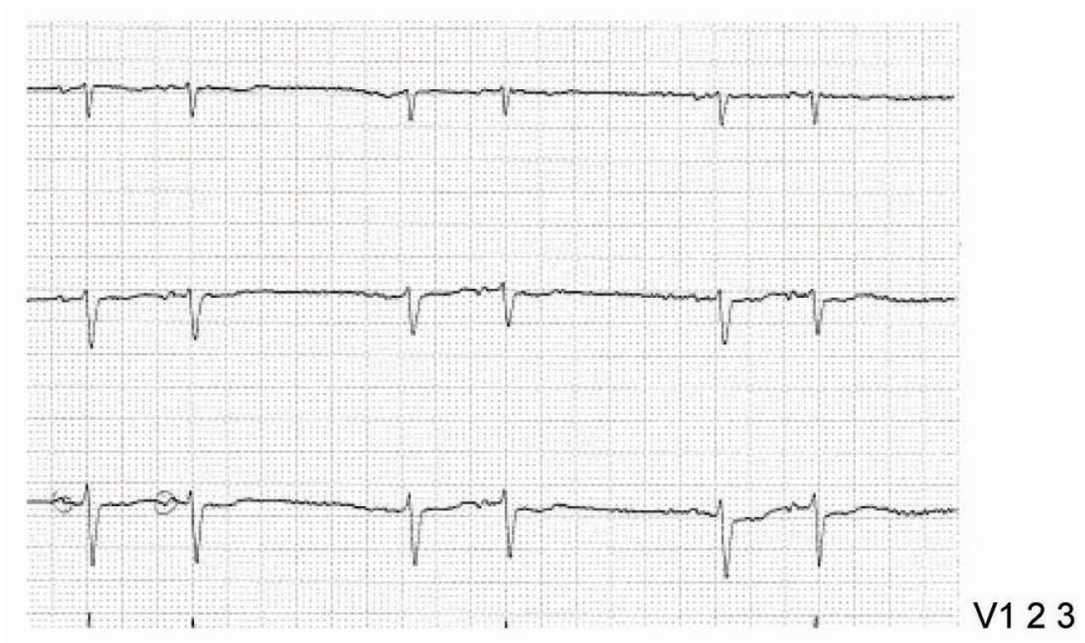

Figure 1. V1-3 leads of the patient's ECG showing atrial bigeminy. The two different morphologies of $p$ initiated from the sinus node and the extranodal focus are encircled.

originating from an ectopic focus has an initial oppression followed by elevation). This was a new finding, as an ECG done 3 months earlier was normal.

The sinus impulse is followed by an atrial premature depolarization (APD) which is coupled to the sinus impulse by a constant interval. APD is followed by a normal ventricular depolarization as evidenced by normal QRS, a rhythm known as Atrio-Ventricular Bigeminy or simply Atrial Bigeminy.

The patient underwent radioactive $\mathrm{I}^{131}$ therapy and resumed his T4 replacement therapy a week later. In an evaluation two months later with a PET scan metastatic disease in the lung was discovered. Despite continuation of T4 replacement therapy bigeminy was still present.

\section{Discussion}

Bigeminal rhythms can occur as a result either of premature beats or of conduction blocks [6]. Atrial bigeminy is a rare rhythm disorder characterized by an APD occurring after each sinus impulse followed by a non-compensatory pause (characteristic of APD). The APD usually arises from a single irritable focus within the atria and, thus, premature $\mathrm{p}$ morphology, although different from that of the normal $\mathrm{p}$ wave, remains constant.

An atrial or junctional focus becomes irritable secondary to excess adrenaline from increased sympathetic input from hyperthyroidism, hypoxia, stress or exogenous substances such as caffeine, cocaine and excess digitalis.

Bigeminy is usually a benign condition, but it may rarely precipitate supra-ventricular and ventricular arrhythmias most commonly atrial fibrillation. The management includes correction of predisposing conditions.
The effects of thyroid hormone on cardiac function are well documented. Triiodothyronine, the active form of thyroid hormone, enters cardiomyocyte nuclei and modulates calcium flux, beta-adrenergic-receptor function, and the transcription of various contractile proteins [1, 4]. Both hyperand hypothyroidism can cause changes in cardiac contraction with hyperthyroidism increasing it while hypothyroidism decreasing it. In addition both conditions are associated with cardiac arrhythmias [4]. Severe hypothyroidism can cause ventricular dysrrhythmias, including torsades de pointes [5]. Hypothyroidism is associated with depressed chronotropy, increased peripheral vascular resistance, decreased cardiac contractility and decreased cardiac output and these effects are reversed with correcting the hypothyroid state. The clinical presentation of hypothyroidism-related cardiac effects is not obvious and most patients have few symptoms and signs. Bradycardia and systemic hypertension, with narrow pulse pressure and slightly increased mean arterial pressure, are the most common findings. Occasionally, cardiac function may be further compromised by the development of pericardial effusion, which occurs with severe, longstanding overt hypothyroidism.

Abnormal ECG findings with QT interval lengthening and $\mathrm{T}$ wave flattening or inversion are common. In addition, hypothyroid patients are more prone to ventricular arrhythmias, due to increased electrical dispersion in the myocardium [7].

Patients with differentiated thyroid carcinoma are treated with total thyroidectomy and adjuvant radioiodine ablative therapy is indicated in selected cases [8], followed by long term TSH suppressive thyroxine replacement therapy. These patients are periodically withdrawn from thyroxine for TSH stimulated thyroglobulin measurements and diagnostic iodine 131 scintigraphy. The consequences of these 
episodes of acute hypothyroidism on cardiac function have been investigated in a few studies. In a study of 11 thyroidectomized patients for a differentiated thyroid carcinoma, sympathovagal variability was greater during hypothyroid periods compared to euthyroid periods in the same patient and to healthy control subjects [9]. In another study of 76 thyroidectomized patients, increased autonomic variability in the hypothyroid state compared with euthyroid state was attributed to decreased intracellular catecholamines production [10]. This autonomic instability observed in the acute hypothyroid state may contribute to the various arrhythmias observed.

In conclusion, we present the first case in the English literature of atrial bigeminy associated with short term overt hypothyroidism. Despite thyroid hormone replacement therapy the patient remained in bigeminy, implying that rhythm changes precipitated by thyroid hormone level changes may not always be reversible. Awareness of the increased frequency of various arrhythmias in thyroid carcinoma patients during periods of hypothyroidism would help in the prompt institution of their optimal indicated management.

\section{Conflict of Interest}

We declare that there is no conflict of interest that could be perceived as prejudicing the impartiality of the research reported.

\section{References}

1. Osman F, Gammage MD, Franklyn JA. Thyroid disease and its treatment: short-term and long-term cardiovascular consequences. Curr Opin Pharmacol 2001;1(6):626631.
2. Slovis C, Jenkins R. ABC of clinical electrocardiography: Conditions not primarily affecting the heart. BMJ 2002;324(7349):1320-1323.

3. Gammage M, Franklyn J. Hypothyroidism, thyroxine treatment, and the heart. Heart 1997;77(3):189-190.

4. Tribulova N, Knezl V, Shainberg A, Seki S, Soukup T. Thyroid hormones and cardiac arrhythmias. Vascul Pharmacol 2010;52(3-4):102-112.

5. Ellis CR, Murray KT. When an ICD is not the answer... hypothyroidism-induced cardiomyopathy and torsades de pointes. J Cardiovasc Electrophysiol 2008;19(10):1105-1107.

6. Wang K, Asinger RW, Marriott HJ. Bigeminal rhythms, common and uncommon mechanisms. J Electrocardiol 2007;40(2):135-138.

7. Galetta F, Franzoni F, Fallahi P, Tocchini L, Braccini L, Santoro G, Antonelli A. Changes in heart rate variability and QT dispersion in patients with overt hypothyroidism. Eur J Endocrinol 2008;158(1):85-90.

8. Pacini F, Schlumberger M, Harmer C, Berg GG, Cohen O, Duntas L, Jamar F, et al. Post-surgical use of radioiodine (131I) in patients with papillary and follicular thyroid cancer and the issue of remnant ablation: a consensus report. Eur J Endocrinol 2005;153(5):651-659.

9. Heemstra KA, Burggraaf J, van der Klaauw AA, Romijn JA, Smit JW, Corssmit EP. Short-term overt hypothyroidism induces sympathovagal imbalance in thyroidectomized differentiated thyroid carcinoma patients. Clin Endocrinol (Oxf) 2010;72(3):417-421.

10. Guasti L, Marino F, Cosentino M, Cimpanelli M, Piantanida E, Mainardi LT, Vanoli P, et al. Changes in autonomic modulation to the heart and intracellular catecholamines. A longitudinal study in differentiated thyroid carcinoma during short-term hypothyroidism and thyroid hormone replacement. Horm Res 2007;67(4):171178. 